\title{
Ovarian, Fallopian Tube, and Primary Peritoneal Carcinoma pN1 TNM Finding v8
}

National Cancer Institute

\section{Source}

National Cancer Institute. Ovarian, Fallopian Tube, and Primary Peritoneal Carcinoma pN1 TNM Finding v8. NCI Thesaurus. Code C139960.

Ovarian, fallopian tube, or primary peritoneal carcinoma with positive retroperitoneal lymph nodes only (histologically confirmed). (from AJCC 8th Ed.) 